Meta

Journal des traducteurs

Translators' Journal

\title{
Microdictionnaire avec micro-encyclopédie et illustrations par diapositives pilotées
}

\section{Daniel Gouadec et A. Le Meur}

Volume 30, numéro 4, décembre 1985

URI : https://id.erudit.org/iderudit/002946ar

DOI : https://doi.org/10.7202/002946ar

Aller au sommaire du numéro

Éditeur(s)

Les Presses de l'Université de Montréal

ISSN

0026-0452 (imprimé)

1492-1421 (numérique)

Découvrir la revue

Citer cet article

Gouadec, D. \& Le Meur, A. (1985). Microdictionnaire avec micro-encyclopédie et illustrations par diapositives pilotées. Meta, 30(4), 332-340.

https://doi.org/10.7202/002946ar d'utilisation que vous pouvez consulter en ligne. 


\title{
MICRODICTIONNAIRE AVEC MICRO- ENCYCLOPÉDIE ET ILLUSTRATIONS PAR DIAPOSITIVES PILOTÉES
}

\author{
B. GouAdec et A. Le Meur
}

L'exploitation de microbanques de terminologie semble de nature à autoriser une réévaluation de la conception et des formes d'utilisation de la documentation linguistique-encyclopédique tant dans la pratique de la traduction que dans la formation des traducteurs. La réalisation de microbanques thématiquement homogènes permet, par interconnexions d'une base de données gérant un " dictionnaire ", d'une base de données arborescente gérant une "encyclopédie spécifique " et d'une base d'illustrations, de construire des outils propres à accroître le rendement des démarches de documentation.

\section{BASE MULTICRITÈRE FORMANT DICTIONNAIRE AUTOMATIQUE}

Le modèle des banques de terminologie est celui de la base de données multicritères permettant une recherche documentaire à partir des unités terminologiques ellesmêmes (avec choix de langues; avec ou sans troncatures) ou à partir de combinaisons de descripteurs ou clés conduisant à la consultation d'ensembles de fiches formant unité sectorielle dans les limites d'un domaine. La réalisation de microbanques organisées selon les principes de la base multicritères ne pose d'autre problème que celui des contenus et de leur structuration. Les obstacles rencontrés tiennent à la nature des logiciels disponibles. Nous avons, dans un précédent article, décrit le schéma de conception d'une microbanque de ce type'. L'exploitation du schéma a conduit, depuis, à la réalisation d'une série de microbanques complémentaires.

Les avantages indiscutables que présente la réalisation de microbanques de terminologie n'échapperont pas au traducteur mais il semblerait que ce soit le responsable de la formation des traducteurs (et terminologues ?) qui puisse en tirer le plus grand profit. En effet, la micro-informatique met à la disposition des instituts de formation un outil d'une extrême souplesse et d'exploitation individualisée. Rien n'interdit donc d'introduire, dans les cursus de formation, la réalisation d'un lexique ou dictionnaire automatique par les sujets en formation. On peut alors dégager les apports de la pratique ${ }^{2}$.

1. La microbanque de terminologie autorise la compréhension du fonctionnement de toute banque (mini-/méga-/giga-banque) et son accessibilité permanente en fait l'outil idéal d'entraînement à l'interrogation.

2. La réalisation d'une base multicritère à partir du banal fichier terminologique oblige les sujets à :

- la rigueur de construction d'un thésaurus ;

- la perception des arbitraires (inévitables) dans la construction d'un thésaurus ;

- la maîtrise des catégories gouvernant un champ ou domaine d'activité ou d'expérience :

- une réflexion sur les critères de construction de banques terminologiques en fonction des utilisateurs et utilisations potentiels. 
3. La manipulation de l'outil apporte un renforcement de la pratique de claviers et, d'une manière générale, la maîtrise au moins partielle de cet outil - maîtrise que l'on ne tardera pas à exiger de tout traducteur - dans les limites de procédures pertinentes (éditeur de texte ; fichier; base de données).

Nous nous garderons de nous appesantir sur les avantages de la réalisation de microbanques de terminologie limitées au modèle de la base multicritère. En vérité, la disponibilité et la souplesse de l'outil commandent d'avoir des exigences plus poussées du point de vue des rendements en situation professionnelle et préprofessionnelle.

La base terminologique multicritère apporte, dans une certaine mesure, une structuration des savoirs : la structuration est d'ordre fonctionnel. En effet, l'interrogation d'une microbanque de terminologie portant sur les DISPOSITIFS ET PROCÉDES DE TRANSFORMATION DU LAIT EN POUDRE ${ }^{3}$ à partir d'un terme tel que $C Y$ CLONE conduira, dans l'ordre, à la consultation de toute fiche dont ce terme est l'un des descripteurs puis à la consultation des fiches dont le terme est la vedette ou élément de la vedette. La base balaie ainsi le champ FONCTIONNEL-CONCEPTUEL avant de balayer le champ PHRASÉOLOGIQUE et avant de fournir la définition proprement dite. Indiscutablement, la procédure de consultation pourrait constituer un handicap pour le traducteur "professionnel ". Mais cette même procédure est une procédure d'ENSEIGNEMENT pour le futur traducteur dans la mesure où elle stipule :

a) que la solution d'un problème terminologique ne passe pas exclusivement par l'appariement des termes ou même par la définition avec.appariement de termes ;

b) que tout concept est, par définition, lié à d'autres concepts dans une série de champs conceptuels-fonctionnels et que la relation détermine les pertinences utiles du concept comme les pertinences intrinsèques du concept conditionnent la nature des formats de relations qu'il peut entretenir avec d'autres concepts.

Bref, la consultation de ce type de microbanque multicritère ENSEIGNE qu'il n'est de savoir (culturel-expérientiel OU linguistique) que structuré ou, pour risquer un barbarisme, 'fonctionnalisé'. La consultation-interrogation par clés linguistiques renforce les liaisons absolues dans la structuration des savoirs linguistiques puisqu'elle propose un balayage phraséologique-syntaxique.

Malgré tout, deux problèmes majeurs demeurent posés. Tout d'abord, la structuration des savoirs (culturels-expérientiels et/ou linguistiques) demeure limitée puisqu'il n'y a pas intégration des éléments de savoir à une exploitation thématique. On peut ainsi obtenir, par exemple, la liste des dispositifs ou processus faisant appel à tel concept/ descripteur mais il restera à conduire un effort de documentation complémentaire pour, ainsi qu'on le dit familièrement : " remettre les choses en perspective " ou " mettre tout cela bout à bout ". Par ailleurs, la seule représentation linguistique n'est pas pleinement satisfaisante. Les limitations du champ documentaire et de la nature du vecteur étant connues, nous avons voulu pallier ces carences par deux moyens qui sont $a$ ) l'exploitation d'une base arborescente fédérée à la base multicritère et $b$ ) l'audio-visuel (diapositives) connecté.

\section{LA BASE ARBORESCENTE FÉDÉRÉE À LA BASE MULTICRITÈRE}

Le principe retenu aux fins d'organisation thématique des savoirs a été celui de l'exploitation d'une base arborescente aux fins de présentation complète, structurée, des données relatives aux procédés et dispositifs pertinents. La base arborescente est une mini-encyclopédie traitant le même secteur ou domaine que la base multicritères. 
La consultation de la base arborescente commence par la consultation d'un SOMMAIRE (première page écran) correspondant à une table de matières. Dans le cas de la POUDRE DE LAIT, le sommaire offre :

1. généralités

2. concentration ou évaporation de lait

3. séchage par contact ou par atomisation

4. séchage secondaire et refroidissement de lá poudre

5. agglomération ou instantanéisation

6. stockage ou ensachage

Le choix d'un numéro enclenche le processus de consultation qui, dans la majorité des cas, conduit directement à une forme secondaire de ramification. Le choix du numéro 3 ci-dessus conduit à une page suivante qui propose un nouveau choix :

1. séchage par contact

2. séchage par atomisation

À ce niveau, le choix du $\mathbf{n}^{\mathrm{o}} 2$ offre un troisième niveau de choix :

1. principe et problèmes de qualité du produit

2. séchage direct ou fractionné

3. atomisation proprement dite

Une fois les choix déterminés, le programme affiche les fiches-contenus de l'encyclopédie correspondant au 'point' de référence.

L'utilisateur peut ainsi obtenir la 'section' de la micro-encyclopédie qui l'intéresse ou, s'il ne dispose pas de critères de choix, consulter directement l'ensemble du ' cours'. La consultation des fiches peut s'interrompre à tout instant et l'utilisateur peut, s'il le désire, revenir à une section antérieure ou passer directement à toute autre section.

La constitution de la base arborescente n'exige pas de compétences particulières. $\mathrm{Au}$ stade de la saisie, le logiciel ${ }^{4}$ propose successivement :

titre de la fiche

suite (titre de la fiche faisant suite à la fiche de référence et chaînée à celle-ci dans la consultation)

antécédent (titre de la fiche précédant la fiche de référence et chaînée à celle-ci dans la consultation)

clef : rubrique recevant les descripteurs de la fiche de référence

guide : possibilité d'introduire, en chaîne sur la fiche de référence, une fiche complémentaire recevant des données servant de guide à l'utilisateur (compléments d'information) dite.

page-écran/pages-écran : espace destiné à la constitution de la fiche proprement

Chacune des fiches de données dans la micro-encyclopédie se situe ainsi dans un ensemble ou dans une chaîne formée entre elle et : la fiche précédente, la fiche suivante, l'éventuelle fiche-guide. Seules apparaissent à l'écran, en consultation, les pages de la micro-encyclopédie.

Du point de vue de la formation des traducteurs, le système ainsi conçu offre l'avantage de contraindre à la rigueur du raisonnement dans l'organisation des chaînages mais aussi à la rigueur de sélection et présentation des informations dans la constitution des fiches-données proprement dites. La réalisation de ce type de base arborescente a, de ce point de vue, un rôle formateur.

À ce stage de l'analyse, nous sommes donc en présence de deux bases terminologiques portant sur le même domaine ou secteur. L'une de ces bases est la base multicritère (microbanque); l'autre est la base arborescente (micro-encyclopédie). La consultation peut intervenir DANS l'une ou l'autre base selon que l'on souhaite obtenir des savoirs 
ponctuels (dont la ponctualité est partiellement corrigée par les balayages de champs de la base multicritère) ou, au contraire, obtenir une thématisation-organisation de ces savoirs. En réalité, nous avons DÉLIBÉRÉMENT choisi de faire en sorte que toute consultation intervienne à la fois DANS L'UNE ET L'AUTRE BASES.

La solution permettant la double consultation automatique est la fédération des deux bases par le biais de DESCRIPTEURS ou CLÉS communs. En d'autres termes, les contenus (fiches) de l'une et l'autre bases (du répertoire terminologique et de la micro-encyclopédie) font l'objet d'une INDEXATION par des mots clés IDENTIQUES. Par conséquent, l'interrogation par mot clé MOBILISÉ des données pertinentes à la fois dans la banque de terminologie et dans le répertoire des savoirs thématiques. Pour être plus précis, il n'y a plus seulement balayage de champ lexical-phraséologique ET balayage de champ conceptuel-fonctionnel, il y a aussi RÉINTÉGRATION CONTEXTUELLE complète ou recontextualisation dans l'expérience ou dans le déroulement des processus.

La fédération des deux bases de données (terminologie et savoirs encyclopédiques pertinents) autorise TROIS types de consultation :

1. Une consultation par interrogation sur le terme, avec ou sans troncatures.

Cette interrogation sur le terme permet d'obtenir directement la fiche qui, dans la base multicritère, DÉCRIT les caractéristiques du terme (catégorie/définition/ appariements/clés/etc.).

S'il y a troncature, la consultation fera émerger le balayage des unités phraséologiques dont le terme constitue un élément.

$\mathrm{Si}$, en outre, le terme faisant l'objet de l'interrogation est descripteur d'autres fiches dans la base multicritères, ces autres fiches seront également mobilisées.

2. Une consultation par mot clé ou par combinaison de mots clés. Cette interrogation par mots clés déclenche le balayage de toutes les fiches pertinentes à la fois dans la base multicritère et dans la base arborescente. En d'autres termes, l'interrogateur obtiendra toutes les fiches ' terminologiques' correspondant à la nature de son interrogation (par exemple, toutes les fiches décrites par ÉVAPORATION et EFFET ou toutes les fiches décrites par SÉPARATION mais pas CYCLONE) puis ' débouchera' dans l'arborescence pour retrouver un 'exposé' lui-même pertinent, avec, une nouvelle fois, possibilité de parcourir l'arborescence, soit en la remontant, soit en la descendant.

3. Une consultation commençant dans l'arborescence et débouchant, à tout moment, dans la base standard.

Nous prenons cette fois l'hypothèse 'défavorable' du traducteur qui, connaissant mal le domaine de sa traduction, estime devoir régler des problèmes documentaires dont la portée ne se limite pas aux définitions et appariements de langue à langue. Il débute son interrogation par SOMMAIRE et se voit présenter le menu dans lequel il effectue un choix. Ayant effectué son choix, il parcourt la section pertinente de la microencyclopédie et obtient ainsi une vue générale de la question (un 'état de la question'). L'avantage de la fédération des bases réside dans le fait qu'il peut alors, à tout moment, quitter l'arborescence pour consulter la base terminologique ' pure' et revenir, une fois telle fiche consultée, au point de l'arborescence qu'il avait quitté. $\grave{A}$ titre d'exemple, si l'interrogateur rencontre, dans une fiche décrivant les procédés de séchage par atomisation, le terme BUSE D'ATOMISATION, il lui suffit de taper ce terme directement, de valider la question pour obtenir la fiche pertinente dans laquelle il trouvera une définition et des équivalents de traduction Le processus est identique s'il souhaite effectuer une interrogation plus large à partir d'un concept donnant lieu à exploitation par mot clé ou descripteur. Ainsi, le concept de FLUIDISATION devenant objet d'interrogation en cours de consultation de l'arborescence mobilisera TOUTES les fiches de la base 
multicritère décrites par ce terme. On atteint ici une exploitation maximale de la dyade de bases fédérées avec, comme toujours, retour à la consultation de l'arborescence après épuisement des fiches pertinentes de la base multicritère.

Les rapides schémas de consultation ainsi décrits donnent la mesure des rendements possibles de la fédération de deux bases dont les objectifs et les structures sont complémentaires au seul prix d'une réflexion sur les systèmes d'indexation. On aboutit ainsi à trois niveaux de savoirs pertinents :

a) les savoirs ponctuels

b) les savoirs organisés et structurés par champs ou savoirs fonctionnels

c) les savoirs organisés et structurés par thème avec séquence de déroulement.

Les trois niveaux de savoir étant, dans la gestion du système, étroitement imbriqués, on peut, quel que soit le mode d'interrogation et quel que soit surtout le type de définition ou le niveau de construction raisonnée de la requête, garantir que l'utilisateur obtiendra une documentation homogène et exhaustive.

Le système 'terminologique-encyclopédique' ainsi construit semble nettement plus satisfaisant que la combinaison, sur supports séparés, des 'dictionnaires' et des 'sources de documentation'. Le problème posé, au fond, est celui de la note dans les ouvrages de terminologie comme dans les banques. En effet, quelles que soient les précautions prises (compte tenu des contraintes d'espace du modèle de fiche), les données consignées en terminologie dans la note tendent à demeurer incomplètes dans la mesure où elles ne sont pas ' relationnelles'. Entendons par là que la fiche/donnée terminologique, avec sa note se présente comme un élément fermé ou clos et que, d'autre part, elle ne propose pas d'éléments d'évaluation au traducteur. Dans le système terminologiqueencyclopédique fédérant deux bases, chacune de celles-ci éclaire l'autre. Il en résulte que la note des fiches terminologiques voit se modifier son statut et sa fonction. Ainsi, le contenu de la note se réduit aux indications de normes ou 'standards' (normalisation ; températures standards ; etc.), c'est-à-dire, aux valeurs universelles qui, incidemment, sont proches des valeurs des définitions. Par contre, le rendement de la note s'accroît puisqu'il y a déplacement de cette note vers la structure arborescente-thématique dans laquelle les données 'encyclopédiques' normalement inhérentes à la note terminologique sont accueillies et reçoivent un traitement de CONTEXTUALISATION dans l'expérience ou re-contextualisation dans un schéma de déroulement qui, en outre, propose des sytèmes d'évaluation. Les valeurs conjoncturelles, tout comme les ensembles de présupposés et implications ou comme les hiérarchisations de phénomènes trouvent droit de cité dans la base arborescente qui recense ainsi des savoirs ÉCLAIRÉS par contextes spécifiques.

Le rendement du système terminologique-encyclopédique ainsi conçu dans l'activité de préparation de la traduction a été mis en évidence dans un test de traduction imposant à deux groupes de sujets de niveaux homogènes et équivalents la traduction d'un même texte. L'un des groupes utilisait le seul 'dictionnaire terminologique' ; l'autre utilisait le système terminologique-encyclopédique. Les performances du second groupe étaient, en gain brut, supérieures de près de $50 \%$ à celles du premier groupe. Les résultats du test ne font que confirmer une évidence : la traduction n'est pas un pur problème de définitions et d'équivalences linguistiques; l'infra-textuel pertinent ne s'arrête pas à un ensemble de concepts, il est dans les relations interconceptuelles (que permet de mấtriser l'interrogation de la base multicritère à partir de combinaisons de mots clés) et il est surtout dans la SIGNIFICATION culturelle-expérientielle et l'évaluation ou exploitation de ces relations interconceptuelles - dans leur recontextualisation fonctionnelle dans un champ d'activité et dans leur réintégration au groupe spécifique d'expérience - que permet d'appréhender la base arborescente. 
Ainsi, la construction d'un système terminologique-encyclopédique que nous assimilons ici à un système terminologique par intersection d'un dictionnaire et de ses notes fonctionnelles organisées permet une expansion des fonctions documentaires de la ' banque' et, surtout, un accroissement considérable des rendements des données et de leurs exploitations. Il ne fait guère de doute que la réalisation de pareils systèmes, si elle n'est que difficilement envisageable dans le cadre des grandes banques de terminologie, ne pose nul problème insurmontable dans la perspective d'échanges de disquettes entre traducteurs utilisant des matériels compatibles ou, encore moins, dans la perspective d'une utilisation aux fins de formation des traducteurs. Peut-être trouverions-nous là un premier moyen d'ouverture des éventails de spécialisations des futurs traducteurs ? Ce premier moyen verrait son rendement accru si le système terminologiqueencyclopédique proposait des représentations complémentaires de la représentation linguistique.

\section{AUDIO-VISUEL CONNECTÉ - LA REPRÉSENTATION PAR DIAPOSITIVES}

Une fois assurée la première voie de l'accroissement des rendements de la source de documentation terminologique-encyclopédique que chaque utilisateur peut utiliser à sa convenance sur micro-ordinateur, nous nous sommes interrogés sur les limites des représentations linguistiques. Il apparaît rapidement que, dans les sources de documentation terminologique, la fréquente complexité des notes à caractère encyclopédique est - principalement - due aux limites des représentations linguistiques. Pourquoi ne pas ajouter une dimension complémentaire en exploitant la diapositive ?

Le projet pilote auquel nous nous sommes attaqués visait l'illustration par diapositives du système documentaire relatif à la production de la poudre de lait. Il suffisait de 80 diapositives pour obtenir des illustrations suffisantes, parmi lesquelles 12 schémas avec légendes bilingues. Les diapositives constituent ainsi l'ensemble des représentations non linguistiques accompagnant la consultation des fiches soit dans l'arborescence, soit dans la base de données multicritère.

La connexion de la diapositive au système informatique est résolue dès l'instant où l'on dispose de l'interface pour projecteur. Sur le plan de la gestion, il suffit alors d'inscrire, pour chacune des fiches, les commandes d'affichage des diapositives pertinentes. On se trouve ainsi en présence de trois types de situations :

a) pas de diapositive pertinente

b) une diapositive pertinente

c) plusieurs diapositives pertinentes.

Dans le premier cas, les fiches ne portent aucune commande d'affichage et, lorsque l'utilisateur les consultera, il n'y aura que la seule représentation linguistique du processus ou du dispositif. Dans le second cas, les fiches portent la commande d'affichage de la diapositive pertinente et, lorsque l'utilisateur consultera les fiches concernées, il obtiendra en même temps, sans aucune manipulation de sa part, la projection de la diapositive - cette dernière restant projetée tant que l'utilisateur lui-même le souhaite, ce qui est nécessaire surtout dans le cas de diapo-schéma. Dans le troisième cas, les fiches pour lesquelles existent plusieurs diapos pertinentes portent une succession de commandes d'affichage : le créateur des bases décide d'un ordre de projection et d'une durée d'attente entre deux diapositives illustrant une même fiche. Lorsque l'utilisateur consulte une fiche de ce type, il se voit projeter une séquence de diapositives. Ce dernier point est d'importance considérable dans la mesure où la séquence de projections de diapositives permet une part de mise en scène ou, pour le moins, une organisation précise des illustrations. Ainsi, à titre d'exemple, pour illustrer une fiche décrivant l'atomiseur à turbine d'une installation de séchage du lait, nous proposons cinq diapositives montrant successivement : 
- l'atomiseur et son moteur sortis de l'installation

- le corps de la turbine

- les ailettes de la turbine et les canaux

- la turbine en place dans la tour et son disperseur d'air

- un schéma du transit du lait Les diapositives sont organisées en une séquence qui reflète l'organisation des données dans la fiche décrivant, dans l'arborescence, le principe de l'atomisation par turbine.

Nous avons donc exploité, sous sa forme la plus élémentaire, l'audio-visuel connecté. Précisons que la micro-informatique permet le pilotage de paires de projecteurs de diapositives et l'exploitation de techniques de fondu-enchaîné. Nous n'avons pas maîtrisé ces raffinements techniques mais avons cependant pu constater que l'illustration pilotée par affichage de fiches pertinentes apporte sans aucun doute la recontextualisation absolue : il n'est plus nécessaire de 'se représenter' les appareils, dispositifs, principes ; il est possible de les voir. Bien évidemment, la pertinence de l'illustration par diapositives dépend de la pertinence des éléments de représentation complémentaire (couleur/matériaux/taille/distribution/organigramme/apparence/etc.) mais, quel que soit le domaine traité, la diapositive apporte indéniablement ce que les publicitaires appellent un $\mathbf{r}^{*}$ plus'.

On pourrait imaginer que se substituent aux diapositives des bandes vidéo ou le vidéodisque. Techniquement, pareilles connexions sont réalisables (le vidéodisque étant réservé, par sa capacité de stockage, aux mini- ou macrobanques de terminologie). Lorsque les logiciels de pilotage vidéo seront tels qu'ils permettront de travailler à la trame près, rien n'empêchera de construire des systèmes documentaires prenant appui sur une bande vidéo accompagnée de son ' dictionnaire'. Il semble cependant que, dans cette hypothèse, les coûts de réalisation écarteront quiconque doit se contenter de microinformatique.

\section{CONCLUSION}

Les systèmes terminologiques-encyclopédiques à gestion automatique avec couplage de diapositives sont de réalisation aisée. Le problème réel posé est celui des indexations complémentaires ou croisées des données dans la base multicritère et dans la base arborescente. Une fois ce problème résolu, l'outil ainsi construit présente un rendement accru par rapport aux banques standard. Il nous semble que la diffusion de la micro-informatique risque de conduire, à brève échéance, à une interrogation sur les possibilités de conception de nouveaux( ?) types d'aides à la traduction et à la formation des traducteurs. Cette même diffusion de là micro-informatique risque également de conduire à des interrogations relatives à la configuration des données terminologiques, aux fonctions et effets des types de représentation envisageables, aux types et catégories de données, aux modalités de leur structuration et à leur statut réel. Nous avons simplement souhaité nous poser certaines de ces interrogations et apporter une modeste réponse.

ANNEXE

Types de contenus des bases de données présentées.

TERME : ATOMISATION CENTRIFUGE (n.f.)

SYNONYME(s) : ATOMISATION PAR TURBINE, n.f. (ATOMISATION PAR) SYSTEME ROTATIF

ANGLAIS : WHEEL ATOMIZATION

DISC ATOMIZATION

ROTARY DISC ATOMIZATION 
DÉFINITION : procédé d'atomisation par turbine tournant à très grande vitesse à l'extrémité d'un arbre entraîné par un moteur électrique vertical. La turbine est alimentée par un distributeur de concentré.

\section{CLEF : DISPOSITIF / ATOMISATION / PROCÉDÉ / TURBINE / SPRAY / DIS- PERSION / PULVÉRISATION / SÉCHAGE / CONCENTRÉ}

DIAPO : $46 A 1567$

Référence : $A P V$

B : type de fiche présente dans la base arborescente :

TITRE : 32332

SUITE : 323321

ANTÉCÉDENT : 3233

GUIDE :

CLEF : ATOMISATION / BUSE / DISPERSION / SPRAY / TURBINE / TURBINE À CANAUX COURBES / CHAMBRE D'ATOMISATION / SÉCHAGE / AIR OCCLUS / ÉMULSIFICATION/

Le dispositif $\hat{d}$ 'atomisation le plus fréquent est l'atomiseur rotatif.

La turbine de l'atomiseur rotatif (46) produit un effet comparable à celui d'une pompe centrifuge, provoquant une forte aération du concentré. L'aération est d'autant plus importante que le produit est faiblement concentré.

L'aération aboutit à la présence d'air occlus dans les particules de poudre et l'on obtient ainsi des poudres de faible densité et souvent mal formées.

Pour obtenir une granulométrie satisfaisante, on peut utiliser une turbine ḋ canaux courbes assurant un effet de dégazage (élimination d'air ou limitation de la reprise d'air du concentré) ou encore une turbine à balayage de vapeur qui limite l'émulsification du concentré (émulsification correspondant aussi à une reprise d'air).

La turbine est un organe coûteux dont le remplacement est fréquent.

(Seule la partie en italiques est affichée.)

Cette fiche de l'arborescence est précédée d'une fiche présentant un choix d'informations sur l'atomisation $(1=$ techniques $2=$ dispositifs $3=$ problèmes techniques et de sécurité) et fait suite au $\mathrm{n}^{\circ} 2$ de ce choix. Elle est suivie, directement, en consultation, d'une fiche décrivant les avantages de l'atomisation centrifuge qui est elle-même suivie d'une série de fiches décrivant des variantes de l'atomisation centrifuge et comportant les inconvénients et avantages de ces variantes. Le chiffre est celui du numéro de diapositive se projetant à l'affichage de la fiche de référence.

\section{C : Diapositives}

Les diapositives sont, en majorité, des photographies d'appareils ou parties d'appareils. Cependant, les diapositives les plus importantes représentent des schémas et diagrammes dont la projection permet à l'utilisateur de situer constamment le lieu et le moment du processus dans lequel s'inscrit son objet de documentation. Ainsi, une diapositive fréquemment affichée donne un schéma général des ÉTAPES de la transformation du lait en poudre. Pour chacune des sous-étapes, nous avons prévu une diapositive schématisant l'opération concernée.

\section{Notes}

1. Voir META.

2. Les apports décrits ci-dessous sont apparus au terme d'une expérience au cours de laquelle des étudiants de licence et maîtrise (langues étrangères appliquées) de l'Université de Rennes 2 ont été amenés à construire une microbanque de terminologie. 
3. Microbanque de terminologie réalisée en 1983-1984 à l'Université de Rennes 2 : comporte une base 'dictionnaire' de 350 fiches indexées et illustrées par diapositives, plus une base 'encyclopédie' de 53 fiches indexées et illustrées par diapositives, plus une base 'iconographique' de 80 diapositives. L'ensemble a été réalisé avec la collaboration du Centre de recherches et d'applications des technologies nouvelles de Rennes et des laiteries PREVAL de Pontivy et Montauban de Bretagne. Le système se trouvait en démonstration sur le stand FRANTERM à EXPOLANGUES 84 à Paris.

4. Le logiciel utilisé est le logiciel ARGUS réalisé par A. Le Meur (CATEN — rue du Clos Courtel ; 35100 Rennes). 